\title{
The effect of cognitive-behavioral consultation on marital adjustment among women referring to health centers of Hamadan in 2015-2016
}

\author{
AREZOO SHAYAN ${ }^{1, A-F}$, MARYAM GAROUSIAN ${ }^{2, A-E}$, MOHAMAD REZA HAVASIAN ${ }^{3, E}$, \\ ORCID ID: 0000-0001-9968-2256 \\ NARGES BABAKHANI 4, A, B, MASOUMEH TARAVATI ${ }^{4, ~ A, ~ B, ~ J A V A D ~ F A R A D M A L ~}{ }^{5, c}$, \\ SEYEDEH ZAHRA MASOUMI ${ }^{4, A-G}$, SOMAYEH KHANI ${ }^{1, \text { B }}$, MINA MASAHI ${ }^{1, \text { B }}$
}

${ }^{1}$ School of Nursing and Midwifery, Chronic Diseases (Home Care) Research Center, Hamadan University of Medical Sciences, Hamadan, Iran

${ }^{2}$ Supervisor Education, Fatemieh Hospital, Hamadan University of Medical Sciences, Hamadan, Iran

${ }^{3}$ Student Research Committee, Alborz University of Medical Sciences, Karaj, Iran

${ }^{4}$ Department of Midwifery, Mother and Child Care Research Center, School of Nursing and Midwifery, Hamadan University of Medical Sciences, Hamadan, Iran

${ }^{5}$ Department of Biostatistics, School of Public Health, Hamadan University of Medical Sciences, Hamadan, Iran

A - Study Design, B - Data Collection, C - Statistical Analysis, D - Data Interpretation, E - Manuscript Preparation, F - Literature Search, $\mathbf{G}-$ Funds Collection

Summary Background. Marital adjustment is one of the most extensive concepts to determine and indicate the level of happiness and stability of the relationship between couples. It influences many aspects of personal and social life.

Objectives. The main objective of this study is to survey the effect of cognitive-behavioral consultation on marital adjustment among women.

Material and methods. The present study was a randomized clinical trial consisting of two groups (an intervention and a control group) with a pre-test and post-test design. 198 qualified married women referred to health centers of Hamadan in 2015-2016. For data collection, the researcher employed a demographic information questionnaire and the Locke-Wallace Marital Adjustment Scale, which were analyzed using descriptive-analytical statistics (covariance), the $t$-test and regression through SPSS 21.0 Software.

Results. The mean score of marital adjustment in the control group was $57.78 \pm 22.63$ and $56.57 \pm 20.32$ before and after the intervention, respectively $(p=0.033)$. In the intervention group, the mean score of marital adjustment was $51.87 \pm 20.23$ and $70.4 \pm 19.47$ before and after the intervention, respectively $(p=0.001)$.

Conclusions. According to the principles of the cognitive-behavioral approach, this approach, along with training about improving communication skills and resolving conflicts between couples, led to a decrease in dissatisfaction and an increase in their adjustment. Key words: behavior, referral and consultation, women.

Shayan A, Garousian M, Havasian MR, Babakhani N, Taravati M, Faradmal J, Masoumi SZ, Khani S, Masahi M. The effect of cognitive-behavioral consultation on marital adjustment among women referring to health centers of Hamadan in 2015-2016. Fam Med Prim Care Rev 2020; 22(2): 152-157, doi: https://doi.org/10.5114/fmpcr.2020.92509.

\section{Background}

In today's ever-changing society, marriage is a complicated process. Social security, love, survival of the generation, spiritual needs and sexual desire are some factors that stimulate people to get married $[1,2]$.

One of the most essential factors in marriage is marital adjustment. It is a situation in which couples feel satisfied with one another in most cases. Adjustment in marriage occurs through making love, taking care of others, accepting each other, understanding each others and satisfying the needs of each other [3-5]. Compatible couples are those who have a high level of agreement with one another. They are satisfied with the type and level of their relationship and with the type and quality of their free time, which help them manage their time and financial issues well [6]. On the one hand, marital adjustment affects many aspects of the individual and social life of humans; it helps with the basis for the proper functionality of the family and the parents' roles [7]. It also increases a couples' life expectancy, health, economic development and life satisfaction [8]. The inconsistencies of marital incompatibility lead to social and behavioral deviations and the decline of moral and ideological values between couples [9].

To ensure marital adjustment, it is necessary that one establish and proceed to effective communication. This requires sharing thoughts, feelings, positive feedback and appreciation [10]. Possession or lack of communication skills affect the state of marital adjustment. Communication without necessary specifications and vague or conditioned communications cause maladjustment between couples. Being involved in or not ending previous relationships can also affect the status of marital adjustment [11]. Marital adjustment is a process that forms during a couple's life, as it requires conformity of tastes, personality traits, the establishment of behavior rules and the formation of interactional patterns. The early days of married life are highly significant. Therefore, marital adjustment is an evolutionary process between couples [8]. 
According to the results of different studies, family environment, attachment style and irrational thoughts are correlated with marital adjustment [12]. There are numerous approaches for incompatible couples in couples' therapy. The cognitivebehavioral method is one of the most important approaches, whose theoretical basis is a combination of the theories proposed by Lieberman, Stuart, Jacobson, Margolin, Guttman, Patterson, Bernstein, Beck and Ellis. This approach attributes marital issues, e.g. lack of intimacy, maladjustment and sexual problems, to the couple's inefficient skills in establishing communication, inability to solve problems and conflicts, irrational expectations and beliefs and negative behavioral exchanges [13]. While treating couples, cognitive-behavioral therapies come after increasing reinforcements and positive interactions, teaching communication skills, solving problems and conflicts and changing destructive thinking patterns [14]. In a study, Toghyani employed cognitive-behavioral education to examine its effect on a group of women and their spouse and their marital satisfaction in Isfahan, Iran. The statistical population of that study comprised all parents whose children attended primary schools in Isfahan. The results of that study showed that marital satisfaction in the experimental group of women who participated in the group sessions of cognitive-behavioral education was higher than the control group after the study and in the follow-up stage [8].

The researcher in the present study believes that it is essential to concentrate on the importance of the relationship between husband and wife, the lack of sufficient information in the field of sex education to women and helping them solve their problem. The present study was conducted to examine the effect of cognitive-behavioral consultation on marital adjustment among women who had referred to health centers in Hamadan due to the high importance of marital adjustment.

\section{Objectives}

This study was conducted to evaluate the effect of cognitive-behavioral consultation on marital adjustment among women.

\section{Material and method}

\section{Design of study method, setting}

This study was a clinical randomized trial and consisted of two intervention groups $(n=99)$ and was conducted on quali- fied women who had referred to health care centers of Hamadan in 2015-2016 and after their informed consent had been obtained.

Sampling method:

The sample size was calculated using the formula

$$
n=\frac{\left(Z_{1-\frac{\alpha}{2}}+Z_{1-\beta}\right)^{2}\left(S_{1}^{2}+S_{2}^{2}\right)}{(d)^{2}}
$$

where $S$ is standard deviation and indices 1 and 2 referring to the intervention and control groups were both equal to $16.1 \mathrm{ac}-$ cording to reference [12], $d$ was equal to 10 and $Z$ indicated percentile of normal distribution which was calculated for indices 0.975 and 0.80 . Based on this information, the sample size was determined to be 82 in each group, and the final sample size was calculated as 198 considering a $20 \%$ loss.

\section{Participants}

The study inclusion criteria included: 1 . Women within an age range of 15-45 (reproductive age); 2. Literacy; 3. At least six months of married life; 4 . Accommodation in Hamadan city; 5. Absence of remarkable physical and psychological diseases, such as schizophrenia and severe depression; 6 . No addiction to drugs or alcoholic drinks. The study exclusion criteria included pregnancy during the study and unwillingness to continue participating in this study. Six health centers were randomly selected. Three centers were assigned to the intervention group and three to the control group. These centers were similar in social, economic, cultural and geographical terms (Figure 1).

Individuals in each group were selected through public recall and by meeting the inclusion criteria and based on the random. All of the participants were informed that participation in the study was completely voluntary. They consented to participate in the study. All of the participants took a pre-test. In the pre-test, the participants of both groups filled out the demographic information questionnaire and Locke-Wallace Marital Adjustment Scale. After completion of the questionnaires, individuals with a total sexual performance score $<100$ participated in the study. The intervention group was provided with consultation, while the control group received conventional care. After intervention and follow-up, both groups took the post-test. Finally, the pre-test and post-test scores were evaluated.

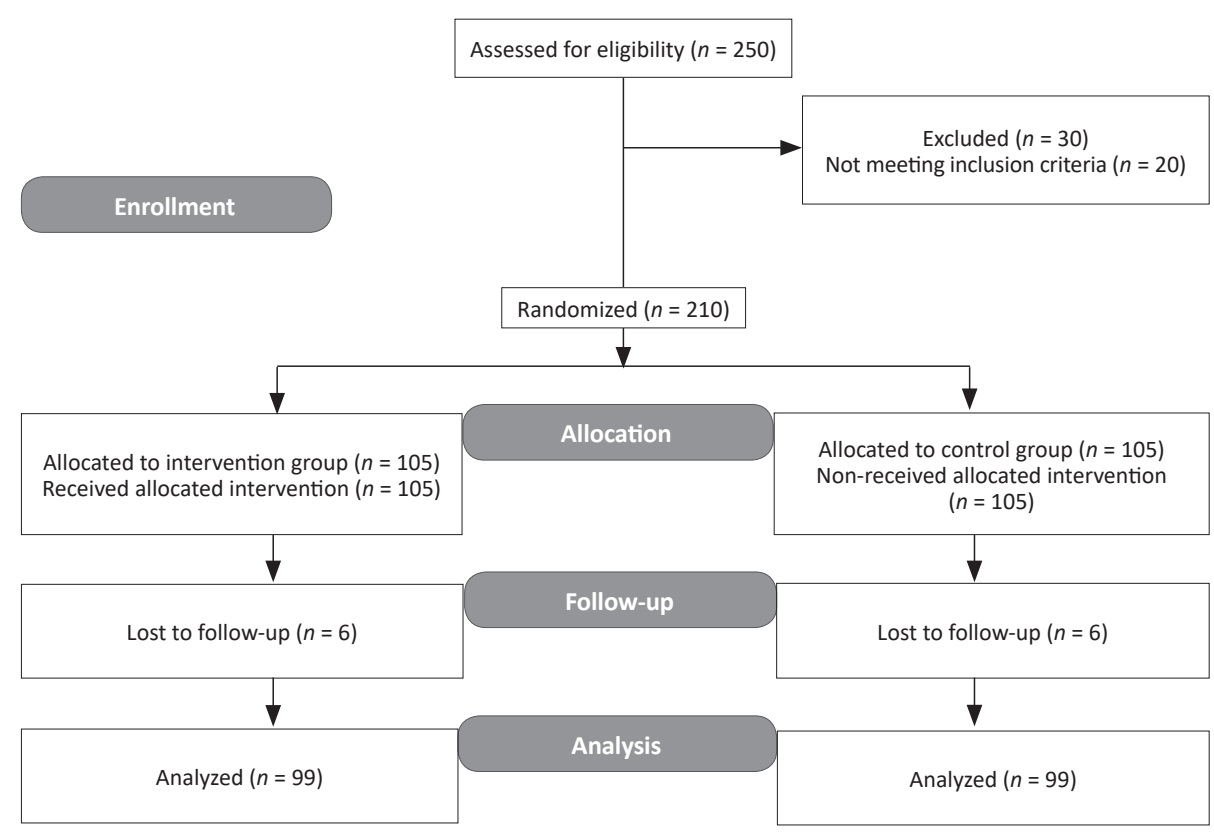

Figure 1. The process of selecting samples 


\section{Intervention}

In this study, consultation sessions was conducted by a cognitive-behavioral approach over 4 two-hour sessions throughout 4 weeks. Counseling was carried out in groups with a maximum of ten people in each group. Each session consisted of questions and answers, lecturing, group discussion and presentation of teaching slides. The content of the training sessions is given in Table 1. The intervention group was informed about the date of attending the sessions through a phone call. Moreover, one day before the sessions, the individuals were reminded of the sessions to prevent sampling loss as much as possible.

\section{Table 1. Educational content of counseling sessions}

Session 1: Identifying inefficient beliefs and explaining negative thoughts regarding sexual satisfaction and marital quality Psychological training: Examining the cognitive-behavioral model and introducing cognitive distortion regarding sexual dissatisfaction and unfavorable marital quality

Homework: Revising cognitive distortion

Session 2: Examining the homework

Psychological training: Examining the methods to fight against cognitive distortion

Homework: Practicing identification of cognitive distortion using sheets for recording thoughts

Session 3: Examining the homework

Psychological training: Introducing coping and prevention methods of behaviors and thoughts leading to sexual dissatisfaction Homework: Cognitive reconstructing, completing the sheets for recording thoughts, practicing coping and prevention of inappropriate behaviors and thoughts

Session 4: Examining the homework

Psychological training: Discussing and examining the factors, prevention approaches, returning from sexual dissatisfaction, increasing sexual satisfaction and improving marital quality

Homework: Practicing prevention approaches to deal with the returning from sexual dissatisfaction, increasing sexual satisfaction and improving marital quality

The consultation was conducted by a researcher who had received the necessary training in these issues under the supervision of a psychiatrist. By the end of the $4^{\text {th }}$ session, both groups participated in the post-test, in which the data of marital adjustment was reconsidered. After the study ended, the control group was taken to a class regarding sexual issues and provided with an educational pamphlet and booklets.

\section{Study instruments}

The researcher employed a demographic information questionnaire to collect the required demographic data and the Locke-Wallace Short Marital Adjustment Scale to measure the participants' marital adjustment. This scale is a short self-report questionnaire designed by Locke and Wallace in 1959 for measuring the quality of marital functioning. It is the most common method for the interpersonal understanding of marriage. It measures the couples' success in activities involved with marital adjustment through 15 questions. The first question is a 7-point Likert scale with scoring 0, 2, 7, 10, 20, 25 and 35 (very satisfied, satisfied and very dissatisfied). One selects either of the options based on the level of happiness, satisfaction with marriage and all other considerations. Questions 2 to 9 are based on a 6-point Likert scale from "I always agree" to "I always disagree", scored $0-5$, which are on different issues. The remaining 6 questions are about the How Couples Decide on Some Marital Issues and the individual's feelings about their spouse. Questions 11,13 and 15 are based on a 4-point Likert scale, and questions 10 and 14 are based on a 3-point Likert scale. Question 12 is based on a 2-point Likert scale, and if both wife and husband select "staying in the family", the score will be 10; if both prefer to "quit the family", the score will be 3; if they have different preferences, the score will be 2 . The scale score is equal to the sum of all scores, ranging from 2 to 158 . An average of over 100 indicates satisfaction with marriage, and an average below 100 shows distress.

Mazaheri used the split-half method and reported a high reliability and validity of $90 \%$. This questionnaire also has high reliability to show the clear distinction between distressed and non-distressed spouses (cited in Nasehi et al.). Sanaeei reported a similar estimation using the Spearman-Brown test and a very genuine correlation coefficient of $90 \%$ between the two group [15]. There was a positive and significant relationship between this questionnaire and the Marital Satisfaction Index Scale, standardized by Sanaeei, which was utilized to measure convergent validity $(r=0.66, p<0.01)[16]$.

\section{Ethical consideration}

The study protocol was approved by the Ethics Committee of Hamadan University of Medical Sciences (IR.UMSHA. REC.1395.50). The protocol was also registered in the Iranian Registry for Randomized Controlled Trials (IRCT201610209014N124) and was conducted after obtaining the permission of the Deputy of Hamadan University of Medical Sciences. This article is the result of a Master's thesis on midwifery counseling.

\section{Statistical analysis}

In the present study, Cronbach's Alpha was used to evaluate internal stability, which was 0.64 . The researcher employed covariance analysis or change analysis so as to analyze the collected data and independent sample $t$-test. Both groups were similar in terms of variables, such as education level, age range, marriage duration and other demographic characteristics. All tests were conducted at a confidence level of $95 \%$. It should be noted that the significance value was set at $5 \%$.

\section{Results}

\section{Findings related to socio-demographic characteri- stics}

Based on the collected data, the mean age in the intervention group and the control group was $35.04 \pm 7.91$ and 32.58 \pm 7.54 years, respectively. There was no notable difference between the two groups, and they were homogenous in terms of education, the spouse's education and the couple's addiction (Table 2).

\begin{tabular}{|c|c|c|c|c|}
\hline \multicolumn{2}{|l|}{ Variables } & Intervention group & Control group & $p$ \\
\hline \multicolumn{2}{|c|}{ Age (years), Mean (Standard Deviation) } & $35.04(7.91)$ & $32.58(7.54)$ & 0.02 \\
\hline \multirow{4}{*}{$\begin{array}{l}\text { Education } \\
n(\%)\end{array}$} & elementary & $11(11.1)$ & $8(8.1)$ & \multirow[t]{4}{*}{0.89} \\
\hline & guidance school & $10(10.1)$ & $16(16.2)$ & \\
\hline & high school & $59(59.6)$ & $63(63.7)$ & \\
\hline & academic and higher education & $22(22.2)$ & $9(9.1)$ & \\
\hline
\end{tabular}




\begin{tabular}{|c|c|c|c|c|}
\hline \multicolumn{2}{|l|}{ Variables } & Intervention group & Control group & $p$ \\
\hline \multirow{4}{*}{$\begin{array}{l}\text { Husband's education } \\
n(\%)\end{array}$} & elementary & $7(7.1)$ & $10(10.1)$ & \multirow[t]{4}{*}{0.59} \\
\hline & guidance school & $14(14.1)$ & $17(17.2)$ & \\
\hline & high school & $56(56.6)$ & $64(64.7)$ & \\
\hline & academic and higher education & $23(23.2)$ & $8(8.1)$ & \\
\hline \multirow{2}{*}{$\begin{array}{l}\text { Spouse addiction } \\
n(\%)\end{array}$} & yes & $26(26.3)$ & $21(21.2)$ & \multirow[t]{2}{*}{0.404} \\
\hline & no & 73 (73.7) & 78 (78.8) & \\
\hline \multirow{2}{*}{$\begin{array}{l}\text { Addiction } \\
n(\%)\end{array}$} & yes & $1(1)$ & $1(1)$ & \multirow[t]{2}{*}{1.00} \\
\hline & no & 98 (99) & $98(99)$ & \\
\hline
\end{tabular}

\begin{tabular}{|c|c|c|c|c|c|c|c|c|c|}
\hline \multirow[t]{2}{*}{ Group } & \multicolumn{4}{|c|}{ Before intervention } & \multicolumn{4}{|c|}{ After intervention } & \multirow[t]{2}{*}{$p^{* *}$} \\
\hline & mean & SD & $\min$ & $\max$ & mean & SD & $\min$ & $\max$ & \\
\hline Control & 57.78 & 22.63 & 19 & 113 & 56.57 & 20.32 & 21 & 109 & $<0.033$ \\
\hline Intervention & 51.87 & 20.23 & 23 & 101 & 70.4 & 19.47 & 33 & 114 & $<0.001$ \\
\hline${ }^{*} p$ & \multicolumn{4}{|l|}{0.054} & \multicolumn{4}{|l|}{0.001} & \\
\hline
\end{tabular}

*T-independent, ${ }^{* *}$ t-test.

\begin{tabular}{|c|c|c|c|c|c|c|c|c|}
\hline \multirow{3}{*}{$\begin{array}{l}\text { Marital adjustment } \\
\text { status }\end{array}$} & \multicolumn{4}{|c|}{ Intervention } & \multicolumn{4}{|c|}{ Control } \\
\hline & \multicolumn{2}{|c|}{ Before intervention } & \multicolumn{2}{|c|}{ After intervention } & \multicolumn{2}{|c|}{ Before intervention } & \multicolumn{2}{|c|}{ After intervention } \\
\hline & $n$ & $\%$ & $n$ & $\%$ & $n$ & $\%$ & $n$ & $\%$ \\
\hline Adjustment & 1 & $1 \%$ & 9 & $9.1 \%$ & 4 & $4 \%$ & 2 & $2 \%$ \\
\hline Maladjustment & 98 & $99 \%$ & 90 & $90.9 \%$ & 95 & $96 \%$ & 97 & $98 \%$ \\
\hline
\end{tabular}

\section{Intervention effect}

The mean score of marital adjustment in the control group was $57.78 \pm 22.63$ and $56.57 \pm 20.32$ before and after the intervention, respectively $(p=0.03)$, which indicated a significant difference. The mean score of marital adjustment in the intervention group rose from $51.87 \pm 20.23$ before the intervention to $70.4 \pm 19.47$ after the intervention, which proved a significant difference $(p<0.001)$ (Table 3$)$.

According to the results obtained for the marital adjustment status of the study groups, the number of the compatible individuals increased from 1 to 9 in the intervention group, but from 4 to 2 in the control group and not increased. Moreover, the incompatible individuals, in terms of marital issues, decreased from 98 individuals (99\%) to 90 individuals (90.9\%) and increased from $95(96 \%)$ to $97(98)$ in the control group, which indicated a significant difference $(p<0.001)$. The number of the compatible individuals increased from 1 to 9 in the intervention group and decreased from 4 to 2 in the control group (Table 4).

\section{Discussion}

Our study aimed to examine the effect of cognitive-behavioral consultation on marital adjustment among women referred to Hamadan health centers. The results indicated that consultation by the cognitive-behavioral approach was remarkably effective in marital adjustment. Basically, the studies mentioned further on are in line with our study. The study of Tehrani et al. showed that interventions related to group training based on Lazarus' multimodal approach [17] can enhance the dimensions of a reciprocal agreement, marital satisfaction and effectual expression in marital adjustment among women. The study conducted by Diner, Lucas and Scollon showed that the number of compatible individuals increased from 1 to 9 in the intervention group [18]. In the control group from 4 to 2, which indicates the effect of consultation on marital adjustment among the women. The results of the study performed by Fagherpor showed that preventive programs based on the cognitive-behavioral approach had a remarkable effect on marital adjustment [19]. Furthermore, Giblin's study [20] indicated the effectiveness of relationship enhancement and couple communication programs. Moreover, the results of the studies carried out by Markman [21] and Garland [22] are in line with this finding. In their study conducted to examine the role of preventive programs and enhancing marital intimacy, Heidarnia et al. found a significant difference in the increase in marital intimacy and its dimensions among the experimental group's couples, which resulted in an increase in marital adjustment [23]. Their finding are in agreement with those of the present study. Markman [21] and Garland's study [22] showed the effect of preventive programs in the form of a "prevention and relationship enhancement program" and communication training. A part of the results of their studies was related to a change in individuals' attitudes as a result of implementing the training-counseling program. Although in literature the issues of knowledge, attitude and behavior were not severally studied, and more attention has been paid to the effect of training on marital compliance and satisfaction and change in attitude, implementing training programs has also been effective in changing individuals, and this finding is significant, as it shows that the individuals' performance in marital life and family can be improved, and in fact, formation of different preventive approaches [23, 24]. According to numerous studies that have been conducted in this field, and also due to their positive results on an increase in marital adjustment and intimacy, conducting this research seemed necessary $[25,26]$. The researcher hopes that the findings will enhance marital adjustment between couples.

\section{Limitations of the study}

In this study, the duration of the follow-up was short, which can be considered a limiting point. 


\section{Conclusions}

As mentioned before, the results of the present study and other studies focusing on cognitive-behavioral training indicated an improvement in marital adjustment among women. Therefore, providing precise counseling training by educated psychologists and midwives and creating interest among women with sexual dysfunction are effective in enhancing marital adjustment among these patients. There is no doubt that counselors and other colleagues that are in touch with such individuals also play an important role in the effectiveness and continuation of providing such services in society. It is also necessary to pay more attention to cognitive-behavioral training due to its positive effects on increasing marital adjustment among couples.

Acknowledgements. The researchers express their gratitude to the Council and Deputy of Research and Technology of Hamadan University of Medical Sciences, the Head of Chronic Disease Care Research Center of Hamadan University of Medical Sciences, the personnel working in health centers in Hamadan and all those who contributed to the present study.

Source of funding: This study was developed using funds provided by Hamadan University of Medical Sciences (No. 950304955).

Conflicts of interest: The authors declare no conflicts of interest.

\section{References}

1. Bahramian J, Bahramian S. The role of psychological hardiness and forgiveness in prediction of marital adjustment. IJCLS 2014; 4(9): 6822-6826.

2. Shayan A, Masoumi Z, Ataollahi M. Investigation relationship between Body Mass Index and general health of patient in Hamadan Fatemieh Hospital, Iran. Der Pharma Chemica 2016; 8(19): 554-558.

3. Naderiasar $\mathrm{MH}$, Hakimi S, Firouz K, et al. Investigation about the relationship between sexual satisfaction and sexual schema with marital satisfaction in women employed in Qazvin Education and Training Office. Journalsih 2014; 2(11): 224-229.

4. Shayan A, Forouhari S, Ahmadinia H. The effect of body mass index on sexual function. Res J Pharm Biol Chem Sci 2015; 6(6): 815.

5. Masoumi SZ, Garousian M, Khani S. Comparison of quality of life, sexual satisfaction and marital satisfaction between fertile and infertile couples. Int J Fertil Steril 2016; 10(3): 290-296, doi: 10.22074/ijfs.2016.5045.

6. Soleimani B, Pasha GH, Eftekhar R. Compared happiness and quality of life in individuals with lower and higher marital satisfaction among married students. Int J Phys Beh Res 2015; 4(1): 58-65.

7. Seyyed Moharrami I, Ghanbari Hashem Abadi B, Asghari E. The effect of solution-focused group counseling on family function and marital satisfaction. Biannual Journal of Applied Counseling 2015; 5(1): 23-40.

8. Abedi B. A survey on the effect of group couple training based on cognitive-behavioral approach on increasing marital adjustment. Quarterly J Sabzevar Univ Med Sci 2015; 21(6): 1055-1063.

9. Mirzaei R, Karimi KH, Shahryari P. The effect of women's employment on marital satisfaction of men. J Health Care 2015; 4(16): 61-69.

10. Shahrestany M, Doustkam M, Rahbarda H. Predicting marital satisfaction over the relational belief and marital intimacy variables in normal and divorced women in Mashhad. J Scientific Research Woman \& Culture 2013; 14(4): 9-17.

11. Bahari F, Fatehizadeh M, Ahmadi SA. The effect of hope, forgiveness and combined marital counseling on interpersonal cognitive distortions of divorcing couples. J Behavioral Sciences Research 2011; 1(8): 25-33.

12. Halford WK, Lizzio A, Wilson KL. Does working at your marriage help? Couple relationship, self-regulation and satisfaction in the first 4 years of marriage. J Fam Psychol 2007; 21(2): 185-194.

13. Pyrflk M, Sudanese M, Shafiabadi A. The Efficacy of Group Cognitive-Behavioral Couple's Therapy (GCBCT), on the decrease of Couple Burnout. Journal of Counseling and Psychotherapy Family 2014; 2(4): 247-267.

14. Mehdipour Bazkiyaei M, Sedaghatfar M. The relationship between marital satisfaction and adjustment spouses intimacy at home. Journal of Analytical-Cognitive Psychology 2015; 3(22): 21-23.

15. Locke HJ, Wallace KM. Short marital adjustment and prediction tests: their reliability and validity. Marriage and Family Living 1959; 21: $251-255$

16. Mehrabi H, Hussainpoor M. Effectiveness of life skills training to increase marital adjustment. J Teach Learn 2010; 1(2): 1-15.

17. Tehrani M, Heidari H, Jafari A. Lazarus multimodal approach was to increase the effectiveness of group dimensions of marital adjustment. JBS 2015; 83-98.

18. Diner E, Lucas R, Scollon CN. Beyond the hedonic treadmill: revising the adaptation theory of well-being. Am Psychol 2006; 61: 305$-314$.

19. Fagherpor M. The effect of a preventive program based on cognitive-behavioral approach to marital fitness. Journal of Women's Health 2010; 3: 16-24.

20. Giblin PS, Sprenkle DH, Sheehan R. Enrichment out came research. A meta-analysis of premarital, marital and Family intervention. Journal of Marital and Family Therapy 2007; 11(3): 257-271.

21. Markman HJ, Renick MJ, Floyd FJ, et al. Preventing marital distress through communication and conflict management training. J Consult Clin Psychol 1993; 61(1): 70-77.

22. Garland DR. Training married couples in listening skills: effects on behavior, perceptual accuracy and marital adjustment. Family Relations 1981; 30(2): 297-306.

23. Heidarnia A, Nazari A M, Soleimanian AA. The effectiveness of prevention programs and improve relations on increasing marital intimacy couples. Journal of Clinical Psychology Studies 2014; 15(4): 61-78.

24. Hahlweg K, Revenstorf D, Schindler L. Effects of behavioral marital therapy on couples' communication and problem-solving skills. Journal of Consulting and Clinical Psychology 1984; 52(4): 553-566, doi: 10.1037/0022-006X.52.4.553.

25. Khodayari Fard M, Hejazi E, Ininezhad N. The effectiveness of Acceptance and commitment consultation (ACT) on self-efficacy and marital satisfaction substance using married women with children. Applied Psychological Research Quarterly 2015; 6(2): 61-75.

26. Khalili A, Shayan A, Khodaveisi M. Construction of professional ethics questionnaire in midwifery. Indian Journal of Forensic Medicine \& Toxicology 2017; 11(2): 237-240.

Tables: 4

Figures: 1

References: 26 
Received: 23.05.2019

Reviewed: 27.06 .2019

Accepted: 16.03 .2020

Address for correspondence:

Zahra Masoumi, PhD, Assoc. Prof.

Mother and Child Care Research Center

Hamadan University of Medical Sciences

Hamadan

Iran

Tel.: +98 8138380150

E-mail: zahramid2001@gmail.com 